\title{
6. Tables of Self-inductance of Circular Coils and of Mutal Inductance of Coaxial Circular Currents.
}

\author{
By Hantaro Nagaoka and Sadazo SakuraI. \\ Institute of Physical and Chemical Research.
}

(Rec. Dec. 28, 1926. Comm. by H. NaGAOKa, Mr.I.A. Jan. 12, 1925.)

In many electromagnetic measurements, there is great need of having tables of selfinductance of circular coils and of mutual inductance of coaxial circles. With the development of radiotelegraphy and telephony, the lack of tables covering large range of dimensions of the coil or of circles was strongly felt. As the formulae for selfinductance and mutual inductance involve elliptic integrals, the evaluation was rather tedious by using Legendre's tables, as the modulus is given in angular measure. To remedy this inconvenience, we have constructed tables ${ }^{1)}$ of elliptic integrals and theta functions with $k^{2}$ as argument, by which many redundant calculations are eliminated. But for practical purposes, tables giving $L$ and $M$ without having recourse to different formulae were much desirable. The present tables were constructed to meet this want and sufficiently accurate to be used in many precise measurements. They will be published in the Scientific Papers of the Institute in the near future.

\section{Selfinductance of Circular Coils.}

One of $\mathrm{us}^{2)}$ has deduced a formula for calculating selfinductance by the formula

$$
L=4 \pi N^{2} \frac{\text { Area of cross section }}{\text { Length }}:=\pi^{2} N^{2} \frac{\text { Diam }^{2}}{\text { Length }} \Omega .
$$

where $\mathbb{R}$ is a constant depending on the ratio of diameter to length and $N$ the number of turns. This seems to be now universally used in calculations of radio works. Denoting the radius by $a$ and the length by $2 l, \&$ can be tabulated with $l / a$ or $a / l$ as argument, as was already given in the paper above cited. The interval was however too coarse, so that in the present table it is given for each 0.001 of the argument, by which the first difference becomes tolerably small. 
At first, tables of $\&\left(k^{2}\right)$, where $k^{2}=\frac{a^{2}}{a^{2}+l^{2}}$ were constructed by using 8-place logarithms according to the formulae

$$
\begin{aligned}
\mathbb{L}\left(k^{2}\right) & =\frac{2}{3 \pi^{2}} \frac{1}{\vartheta_{0}^{\prime 2}(o \mid \tau)}\left\{\frac{\vartheta_{0}^{\prime \prime}(o \mid \tau)}{\vartheta_{0}(o \mid \tau)} \frac{k^{\prime 2}}{k^{2}}-\frac{\vartheta_{2}^{\prime \prime}(o \mid \tau)}{\vartheta_{2}(o \mid \tau)}\right\}-\frac{4}{3 \pi} \frac{k}{k^{\prime}} \\
\mathbb{S}\left(k^{2}\right) & =\frac{4}{3 \pi} \frac{1}{k^{\prime} k^{2}}\left\{k^{\prime 2} K+\left(1-2 k^{\prime 2}\right) E\right\}-\frac{4}{3 \pi} \frac{k}{k^{\prime}}\left(k^{2}=0.490 \text { to } 0.820\right) \\
\mathbb{S}\left(k^{2}\right) & =\frac{4}{3 \pi} \frac{k^{\prime}}{k^{2}}(K-E)+\frac{4}{3 \pi} \frac{\left(1+\vartheta_{11}\left(o \mid \tau_{1}\right)\right)\left(1-\vartheta_{0}\left(o \mid \tau_{1}\right)\right)}{\vartheta_{1}^{2}\left(o \mid \tau_{1}\right)} \\
& +\frac{2}{3 \pi^{3}} \frac{1}{\vartheta_{2}^{2}\left(o \mid \tau_{1}\right)} \frac{\vartheta_{1}^{\prime \prime}\left(o \mid \tau_{1}\right)}{\vartheta_{0}\left(o \mid \tau_{1}\right)} \log \frac{1}{q_{1}} \quad\left(k^{2}=0.820 \text { to } 1.000\right)
\end{aligned}
$$

and interpolated.

In the former table, values of $\mathbb{R}$ with $a / l$ as argument, were given, but in the present, that with $l / a$ was constructed, the range covering $l / a=$ 0.001 to 1 at intervals of 0.001 . This will be found useful for finding selfinductance of short coils.

\section{Mutual Inductance of Coaxial Ciruclar Currents.}

The formula for calculating the mutual inductance was first given by Maxwell'3 in terms of elliptic integrals, but it was found that by using $q$-series, it can be evaluated without having recourse to tables of the integrals, as was shown by one of us. ${ }^{4)}$ The $q$-series are rapidly convergent, so that in the construction of the present table, we have utilised them in different forms.

Denoting the radii of the circles by $A$ and $a$, and the distance by $z$,

and

$$
k^{2}=\frac{4 A a}{(A+a)^{2}+z^{2}},
$$

$$
\begin{aligned}
& \log _{10} \frac{M}{\sqrt{ } A a}=\log _{10} \frac{2}{\vartheta_{2}{ }^{2}(o \mid \tau)}\left\{\frac{\vartheta_{1}^{\prime \prime \prime}(o \mid \tau)}{\vartheta_{1}^{\prime}(o \mid \tau)}-\frac{\vartheta_{2}^{\prime \prime}(o \mid \tau)}{\vartheta_{2}(o \mid \tau)}\right\} \\
& =\log _{10} 16 \pi^{2}+\frac{3}{2} \log _{10} q+m\left(3 q^{4}-4 q^{6}+\frac{9}{2} q^{8}+0+4 q^{12}+\cdots\right)
\end{aligned}
$$

where $m=0.43429448=$ modulus of common logarithm.

$$
\left(k^{2}=0.000 \text { to } 0.970\right)
$$


No. 1.]

Tables of Self-inductance of Circular Coils etc.

$$
\begin{gathered}
\frac{M}{\sqrt{A a}}=\frac{8}{\pi} \frac{1}{\vartheta_{0}{ }^{2}\left(o \mid \tau_{1}\right)}\left\{-\pi^{2}-\frac{1}{4} \operatorname{logn} \frac{1}{q^{1}}\left(\frac{\vartheta_{2}^{\prime \prime}\left(o \mid \tau_{1}\right)}{\vartheta_{2}\left(o \mid \tau_{1}\right)}+\frac{\vartheta_{3}^{\prime \prime}\left(o \mid \tau_{1}\right)}{\vartheta_{3}\left(o \mid \tau_{1}\right)}\right)\right\} \\
\left(k^{2}=0.880 \text { to } 1.000\right)
\end{gathered}
$$

By tabulating $M / A a$ in terms of $k^{2}$, many tediuos calculations are eliminated. As the table is given at intervals of 0.001 in $k^{2}$, there is no difficulty of interpolation, which is much felt in using Maxwell's table."

\section{Force between Coaxial Circular Currents.}

In connection with the table of mutual inductance, we have also constructed that giving the force $\left.{ }^{6}\right)$ between the currents $\frac{\partial M}{\partial z}$, as there is need of evaluating it in designing electromagnetic balances or in the determination of olum. It was also calculated by using $q$-series according to the following formulae.

$$
\begin{gathered}
\log _{10} \frac{\sqrt{A a}}{z} \frac{\partial M}{\partial z}=\log _{10} 192 \pi^{2}+\frac{5}{2} \log _{11} q+m\left(20 q^{2}+25 q^{4}\right. \\
\left.+\frac{20}{3} q^{6}+\frac{15}{2} q^{8}+152 q^{10}+\ldots \ldots\right) \quad\left(k^{2}=0.000 \text { to } 0.910\right) \\
\frac{\sqrt{A a}}{z} \frac{\partial M}{\partial z}=\frac{\pi}{16} \frac{1}{q_{1}}\left\{\left(1+12 q_{1}-192 q_{1}{ }^{2}+1232 q_{1}{ }^{3}-5634 q_{1}{ }^{4}+\ldots . .\right)\right. \\
-12 q_{1} \log n \frac{1}{q_{1}}\left(1-10 q_{1}+60 q_{1}{ }^{2}-300 q_{1}{ }^{3}+1300 q_{1}{ }^{4}-\ldots \ldots\right) \\
\left(k^{2}=0.880 \text { to } 1.000\right)
\end{gathered}
$$

All these tables are given to six places, as 8-place logarithms were utilised in the computations; the last place may sometimes be in error by unity.

Various methods of computation can be suggested, but in the construction of the present table, we had recourse to $q$-series, as the convergence seemed to be most favourable for our purpose, and we possessed already 8-place tables of $q$ with $k^{2}$ as argument. The arithmeticogeometric means may also be utilised as suggested by L. King, ${ }^{7}$ but we have not yet tested the method.

Tables of similar nature have already been published mostly by the Bureau of Standards, Washington, and extensively used. The advantage of the present table would lie mostly in the easiness of interpolation, as the argument is at close intervals, and may be found to be of practical use. 


\section{References.}

1) Nagaoka a. Sakurai: Tables of Theta Functions, Elliptic Integrals $K$ and $E$, and other Functions associated with them, Tokyo, (1922)

2) Nagaoka: Journ. Coll. Sci. 27 (1909) Art. 6. p. 18; Rosa a. Grover; Bull. Bur. Stand. 8 (1912) 119.

3) Maxwell: Treatise on Electricity and Magnetism 2.

4) Nagaoka: Journ. Coll. Sci. 16 (1903) Art. 15 ; Phil. Mag. 6 (1904) 19.

5) Maxwell: loc. cit.

6) Rayleigh: Sci. Papers 2278 ; Rosa, Dorsey a. Miller: Bull, Bur. Stand. 8 (1911) 269; Grover: 12 (1916) 317; Nagaoka: Journ. Coll. Soi. 16 (1903) Art. 15 ; Phil. Mag. 6 (1903) 19 ; Tokyo Phys. Math. Soc. Proc. 6 (1911) 156; 9 (1917) 85.

7) L. King: Direct Calculation of Elliptic Functions and Integrals, Cambridge (1924). 